\title{
A General Complex Variable Boundary Element Method
}

R. J. Whitley, ${ }^{1}$ T. V. Hromadka $\|^{2}$

${ }^{1}$ Department of Mathematics, University of California, Irvine, CA 92697

${ }^{2}$ Failure Analysis Associates, 3187 Redhill Blvd. Suite 100, Costa Mesa, CA 92626

Received 12 April 2000; accepted 1 December 2000

The solution to any 2-dimensional potential problem, with continuous data given on the boundary of a bounded domain with connected complement, can be approximated by sums

$$
\operatorname{Re} \sum c_{n} f\left(\alpha_{n} z+z_{0}\right)
$$

where $f$ is any preassigned non-polynomial analytic function. $(\mathcal{C} 2001$ John Wiley \& Sons, Inc. Numer Methods Partial Differential Eq 17: 332-335, 2001

Keywords: Dirichlet problem, potential, complex variable boundary element method

An important applied problem is the Dirichlet problem for a 2-dimensional potential:

$\Omega$ a bounded plane domain with boundary and the complement of $\Omega \cup \Gamma$ connected

$$
\text { g a given continuous real-valued function defined on } \Gamma \text {. }
$$

Find a function u harmonic in $\Omega$ and continuous on $\Omega \cup \Gamma$ with $u=g$ on $\Gamma$.

The Complex Variable Boundary Element Method (CVBEM) [1] for approximating the solution to this problem involves the numerical determination of parameters so that the analytic function $h$

$$
h(z)=c_{0}+c_{0}^{\prime} z+\sum_{n=1}^{N} c_{n}\left(z-\beta_{n}\right) \log _{\beta_{n}}\left(z \quad \beta_{n}\right)
$$

Correspondence to: Prof. Robert Whitley, University of California Irvine, Dept. of Mathematics, Irvine, CA 92717 (e-mail: rwhitley@math.uci.edu)

(c) 2001 John Wiley \& Sons, Inc. 
satisfies

$$
|\operatorname{Reh}(z)-g(z)|<\epsilon, \text { for } \mathrm{z} \text { on } \Gamma
$$

from which

$$
|\operatorname{Reh}(z)-u(z)|<\epsilon, \text { for } \mathrm{z} \text { in } \Omega \cup \Gamma
$$

follows from the maximum principle. (The subscript on the complex logarithm refers to the branch cut chosen, for which see [2].) The proof that it is possible to approximate the solution by sums of the form (2) is given in [3].

The form of the approximate solution (2) raises the question of the exact role played by the function $f(z)=(z-\beta) \log (z-\beta)$. Can similar approximation results be obtained using other functions? This question is answered here by showing that a surprisingly large class of analytic functions can be used in approximations of the same general type as (2).

The ease with which one of our hypotheses can be satisfied is made clear by an elementary Lemma [4, Ex. 2, page 227].

Lemma 1. Let $f$ be analytic on a ball $U=B\left(z_{1}, \rho\right)=\left\{z:\left|z-z_{1}\right|<\rho\right\}$. If $f$ is not a polynomial on $\mathrm{U}$, there is a point $z_{0}$ in $\mathrm{U}$, where every derivative of $f$ is nonzero:

$$
f^{(n)}\left(z_{o}\right) \neq 0, \mathrm{n}=0,1, \ldots
$$

Proof. Let $D_{n}=\left\{\mathrm{z}\right.$ in $\left.\mathrm{U}: f^{(n)}(z)=0\right\}$. If (5) fails to hold, then $U=\cup\left\{D_{n}: n=0,1, \ldots\right\}$. Then any closed uncountable subset of $U$ intersects at least one $D_{m}$ in an infinite set with a limit point in $\mathrm{U}$; by the identity theorem, $f^{(m)}$ is then identically zero and $f$ is a polynomial.

Note that because the ball in the Lemma can be chosen to have any positive radius less than or equal to the original radius $\rho$, the point $z_{0}$ can be taken to be arbitrarily close to the original center $z_{1}$.

Theorem 2. Consider the Dirichlet problem (1). Let $f$ be analytic on a ball $B\left(z_{0}, \rho\right)$, where it is not a polynomial; using the above Lemma, we can suppose that (5) holds. Then for any given $\epsilon>0$ there is a sum

$$
h(z)=\sum_{n=0}^{N} c_{n} f\left(\alpha_{n} z+z_{0}\right)
$$

that is analytic on $\Omega$ and continuous on $\Omega \cup \Gamma$, with

$$
|\operatorname{Reh}(z)-g(z)|<\epsilon \text { for } \mathrm{z} \text { on } \Gamma \text {. }
$$

Consequently, Reh is a harmonic function that is within $\epsilon$ of the exact solution to the Dirichlet problem.

Proof. Because $K=\Omega \cup \Gamma$ is compact, there is a ball $B(0, R)$ that properly contains $\mathrm{K}$. If $|\alpha|<r=\frac{\rho}{R}, f\left(\alpha z+z_{0}\right)$ is analytic for $\mathrm{z}$ in a neighborhood of $K$.

The space $C(K)$ is the Banach space consisting of all continuous functions on $K$ taken with the supremum norm. Let clP(K) denote the closure of the subspace of all polynomials $\mathrm{P}(\mathrm{K})$ in $C(K)$. For each fixed value of $\alpha$ in $\mathrm{B}(0, \mathrm{r})$, let $w_{\alpha}$ denote the function given by $w_{\alpha}(z)=f\left(\alpha z+z_{0}\right)$. Let

$$
M=s p\left\{w_{\alpha}:|\alpha|<r\right\}
$$

be the vector subspace of $C(K)$ spanned by the indicated set of functions $w_{\alpha}$. Because $w_{\alpha}$ is analytic on a ball containing the compact set $K$, the partial sums of its Taylor's series about zero converge uniformly to it on $K$, which is to say that $w_{\alpha}$ belongs to $\mathrm{clP}(\mathbf{K})$. 
We claim that the closure of $M$, clM, equals clP(K):

$$
c l M=c l P(K) \text {. }
$$

If this is not true, there is a function $Q_{0}$ in $\operatorname{clP}(\mathrm{K})$ whose distance from $\mathrm{M}$ is positive:

$$
\inf \left\{\left\|Q_{0}-g\right\|: \mathrm{g} \text { in } \mathbf{M}\right\}>\delta>0 .
$$

Because $Q_{0}$ belongs to $\operatorname{clP}(\mathrm{K})$, there is a polynomial $P_{0}$ with $\left\|Q_{0}-P_{0}\right\|<\frac{\delta}{2}$, and, thus,

$$
\inf \left\{\left\|P_{0}-g\right\|: \mathrm{g} \text { in } \mathrm{M}\right\}>\frac{\delta}{2} .
$$

By the Hahn-Banach theorem, there is a continuous linear functional $x^{*}$ on $C(K)$ with

$$
x^{*}(M)=0
$$

and

$$
x^{*}\left(P_{0}\right)=1 \text {. }
$$

Because (12) holds, $x^{*}\left(w_{\alpha}\right)=0$ for all $|\alpha|<r$. Because $w_{\alpha}$ has a power series about zero that converges uniformly on $\mathrm{K}$, i.e., in the norm of $C(K)$, the continuity and linearity of $x^{*}$ allow us to write

$$
0=x^{*}\left(w_{\alpha}\right)=\sum_{n=0}^{\infty} a_{n} \alpha^{n} x^{*}\left(z^{n}\right) .
$$

Because the power series (14) in the variable $\alpha$ is identically zero for $\alpha$ in $B(0, r)$, its coefficients must all be zero, and because $n ! a_{n}=f^{(n)}\left(z_{o}\right) \neq 0$, this implies that $x^{*}\left(z^{n}\right)=0$ for $n=$ $0,1, \ldots ;$ hence, $x^{*}\left(P_{0}\right)=0$, contradicting (13).

To complete the proof, the Walsh-Lebesgue Theorem [5, p 173], states that, for the Dirichlet problem (1) above, given $\epsilon>0$ there is a polynomial $P_{1}$ with $\left|\operatorname{Re} P_{1}(z)-g(z)\right|<\epsilon$ holding on $\Gamma$. By what has been shown, the subspace $M$ spanned by the $w_{\alpha}$ is (uniformly) dense in the closure of $\operatorname{clP}(\mathrm{K})$ of the polynomials, and so there is a sum of the form (6) that approximates $P_{1}$ to within $\epsilon$ on $K$, and that, therefore, satisfies $|\operatorname{Reh}(z)-g(z)|<2 \epsilon$ for $z$ on $\Gamma$.

The hypothesis that $f$ not be a polynomial is necessary, for if $f$ is a polynomial of degree $\mathrm{m}$, then the sum (6) is a polynomial of degree less than or equal to m, and (7) cannot hold for all $\epsilon>0$ and all continuous $\mathrm{g}$.

An examination of the proof of the theorem shows that the parameters $\left\{\alpha_{n}\right\}$ appearing in the approximating sum (6) can be restricted to lie in any given infinite subset of $B(0, r)$, which has a limit point in $B(0, r)$.

Under weak hypotheses on the boundary $\Gamma$, the theorem implies that a given boundary value function $\mathrm{g}$ in $L^{p}(\Gamma)$ can be approximated in the $L^{p}(\Gamma)$ norm by a sum of the form (6); see the proof of Corollary 2 in [3].

Note that, if $f$ is analytic on $B\left(z_{0}, \rho\right)$ and (5) holds, the same is true for the derivative $f^{\prime}$ and is also true for any anti-derivative $F$, provided that $F\left(z_{0}\right) \neq 0$.

To establish the connection between the sums (2) and (6), begin by considering the function $\frac{1}{z}$. Two integrations give the function

$$
f(z)=-z+z \log (z),
$$


where $\log (z)$ has as branch cut the nonnegative $\mathrm{x}$-axis. For $z_{0}=1$, the functions

$$
w_{\alpha}(z)=-(\alpha z-1)+(\alpha z-1) \log (\alpha z-1)
$$

are analytic on $\mathrm{B}(0,1)$ for $|\alpha| \quad 1$.

Because

$$
(z-\beta) \log _{\beta}(z-\beta)
$$

and

$$
\beta\left(\frac{*}{\beta} z-1\right) \log \left(\frac{*}{\beta} z-1\right)
$$

both have the same second derivative, it follows that on a common connected domain of analyticity these two functions differ by a linear function of z. If $|\beta|>R$, where $K$ is contained in $\mathrm{B}(0, \mathrm{R})$, then the functions in (17) and (18) will both be analytic on $K=\Omega \cup \Gamma$. Combining all the linear terms into the first two terms, (2) can be written as

$$
a_{0}+a_{0}^{\prime} z+\sum_{n=1}^{N} a_{n}\left(\frac{1}{\beta_{n}} z-1\right) \log \left(\frac{1}{\beta_{n}} z-1\right),
$$

in direct correspondence with (6).

One aspect of the CVBEM approximation that does not follow from the theorem is that the nodes $\left\{\beta_{n}\right\}$ in (2) can be chosen to lie on $\Gamma$, a result that depends on the specific structure of the logarithm and requires the use of curvilinear branch cuts, if $\Omega$ is not convex. This is useful numerically, because more nodes can be placed on the sections of $\Gamma$, where the boundary function $\mathrm{g}$ changes more rapidly.

\section{References}

1. T. Hromadka II and R. Whitley, Advances in the complex variable boundary element method, SpringerVerlag, New York, 1998

2. R. Whitley and T. Hromadka II, Complex logarithms, Cauchy principal values, and the complex variable boundary element method, Appl Math Model (1994), 423-428.

3. R. Whitley and T. Hromadka II, Numerical solutions of the Dirichlet problem via a density theorem, Numer Methods Partial Differential Eq (1994), 369-381.

4. W. Rudin, Real and complex analysis, McGraw-Hill, New York, 1974.

5. T. Ransford, Potential theory in the complex plane, Lond Math Soc, London, 1994. 